\title{
Adult bone marrow-derived cells trans- differentiating into insulin-producing cells for the treatment of type I diabetes
}

\author{
Seh-Hoon $\mathrm{Oh}^{1}$, Toni M Muzzonigro ${ }^{1}$, Si-Hyun Bae ${ }^{1}$, Jennifer M LaPlante ${ }^{1}$, Heather M \\ Hatch $^{1}$ and Bryon E Petersen ${ }^{2}$ \\ ${ }^{1}$ Department of Pathology, Immunology and Laboratory Medicine and ${ }^{2}$ Program in Stem Cell Biology and \\ Regenerative Medicine, College of Medicine, University of Florida, Gainesville, FL, USA
}

\begin{abstract}
Recent findings suggest that bone marrow (BM) cells have the capacity to differentiate into a variety of cell types including endocrine cells of the pancreas. We report that BM derived cells, when cultured under defined conditions, were induced to trans-differentiate into insulin-producing cells. Furthermore, these insulinproducing cells formed aggregates that, upon transplantation into mice, acquired architecture similar to islets of Langerhans. These aggregates showed endocrine gene expression for insulin (I and II), glucagon, somatostatin and pancreatic polypeptide. Immunohistochemistry also confirmed that these aggregates were positive for insulin, somatostatin, pancreatic polypeptide and C-peptide. Also, Western and ELISA analysis demonstrated expression of proinsulin and/or secretion of active insulin upon glucose challenge. Subcapsular renal transplantation of these aggregates into hyperglycemic mice lowered circulating blood glucose levels and maintained comparatively normal glucose levels for up to 90 days post-transplantation. Graft removal resulted in rapid relapse and death in experimental animals. In addition, electron microscopy revealed these aggregates had acquired ultrastructure typically associated with mature beta $(\beta)$ cells. These results demonstrate that adult BM cells are capable of trans-differentiating into a pancreatic lineage in vitro and may represent a pool of cells for the treatment of diabetes mellitus.
\end{abstract}

Laboratory Investigation (2004) 84, 607-617, advance online publication, 22 March 2004; doi:10.1038/labinvest.3700074

Keywords: bone marrow cell; C-peptide; diabetic therapy; insulin; trans-differentiation

Stem cells are self-renewing elements that can generate many cell types in the body and are found in both adult and fetal tissue. Cell therapy using stem cells and their progeny is a promising approach that may be capable of addressing many unmet medical needs. ${ }^{1}$ Recently, stem cell research has quickly progressed, allowing researchers to isolate and purify stem/progenitor cell populations from various tissues (ie hematopoietic, vascular endothelial and neural stem cells, as well as hepatic oval cells). ${ }^{2-4}$ Bone marrow (BM)derived cells have been shown to differentiate into

Correspondence: BE Petersen, Department of Pathology, PO Box 100275, University of Florida, Gainesville, FL, 32610, USA

E-mail: petersen@pathology.ufl.edu

SHO and BEP are inventor/coinventor of a patent(s) related to this technology and may benefit from royalties paid to the University of Florida related to its commercialization.

Received 12 December 2003; revised 10 February 2004; accepted 11 February 2004; published online 22 March 2004 various lineages, such as hepatocytes, in vivo and in vitro. $^{5-7}$ As stem cell research progresses, new methods for the therapy and treatment of diseases such as diabetes mellitus may be possible. In addition, stem cell research may advance our understanding of organ regeneration and the mechanism(s) thereof.

The pancreas is composed of two compartments, the exocrine and endocrine, which have very distinct functions. The endocrine compartment consists of islets of Langerhans, which are composed of clusters of four cell types that synthesize the peptide hormones: insulin ( $\beta$-cells), glucagon $(\alpha$ cells), somatostatin ( $\delta$-cells) and pancreatic polypeptide ( $\gamma$-cells). These cells have been shown to differentiate from ductal epithelial stem cells through sequential differentiation during embryogenesis. ${ }^{8-10}$

Type I diabetes results when the $\beta$-cells of the pancreas are destroyed by T-cells of the immune system, which markedly reduce the number of 
$\beta$-cells resulting in lower insulin production. The worldwide prevalence of Type I diabetes is rapidly increasing and considerable interest has developed in finding mechanisms that may increase active insulin secretion. Various endeavors to solve this challenge have been made through stimulating the endogenous regeneration of islets and through transplantation of donor islets or in vitro-differentiated islet-like cells. ${ }^{11-14}$

Recent reports have described, a multipotent stem cell within the pancreas. ${ }^{15}$ These cells have the capacity to differentiate into pancreatic islet-like structures in vitro. Furthermore, other cell types, such as hepatic oval cells and embryonic stem cells are also capable of differentiating into pancreatic endocrine hormone-producing cells both in vitro and in vivo. ${ }^{16-18}$

Current research, attention has focused on the possible use of the controlled differentiation of stem cells to obtain specialized cells useful in treating many diseases. Here, we show that BM-derived cells can be efficiently induced to differentiate into insulin-producing cells, as well as other cell types, typical of endocrine pancreas. In addition, these cells self-assemble to form functional pancreatic islet-like structures. The insulinproducing cell clusters isolated from BM cells demonstrated reversal of hyperglycemia when implanted into a chemically induced diabetic NOD/ scid mouse model. In addition, these cells acquire ultrastructural characteristics typical of a mature $\beta$-cell phenotype. The data provided may allow for the treatment of diabetes via a stem cell therapy approach.

\section{Materials and methods}

\section{BM Cell Culture}

All procedures regarding animals were conducted according to institutionally approved protocols and guidelines. BM cells were collected from the long bones of F-344 rats (Harlan, Indianapolis, IN, USA). The BM cells were cultured in DMEM supplemented with $10 \%$ FBS. After $60 \mathrm{~min}$ of incubation, nonadherent cells were collected and washed with fresh, serum-free DMEM medium. The cells were replated in serum-free DMEM medium at a cell density of $1 \times 10^{7} / \mathrm{cm}^{2}$ in the presence of $1 \%$ DMSO. The cells were then cultured in DMEM supplemented with $1 \%$ DMSO for 3 days followed by either high $(25 \mathrm{mM})$ or low $(5.5 \mathrm{mM})$ glucose DMEM media supplemented with $10 \%$ FBS for 7 additional days. They were plated in plastic six well plates on slide coverslips $\left(22 \times 22 \mathrm{~mm}^{2}\right)$ coated with $0.3 \%$ type I collagen, which was extracted from the rat-tail tendon by the method described by Michalopoulos and Pitot. ${ }^{19}$

\section{Detection of Transcription Factors, Glut-2 and Pancreatic Endocrine mRNAs}

Total RNA was isolated from the adult rat pancreas and BM cells treated with 1\% DMSO/DMEM low glucose medium (Day 3), DMEM high glucose medium with $10 \%$ FBS (days 7 and 10), or nonculture BM cells by the RNeasy kit (Qiagen, Valencia, CA, USA). A measure of $2 \mu \mathrm{g}$ RNA was used for cDNA synthesis. RT-PCR was performed as previously described by Yang et al. ${ }^{16}$ The resulting products were amplified, electrophoresed in $1.5 \%$ agarose gels containing EtBr, and purified by gel elusion. These products were directly sequenced using an AmpliTaq cycle sequencing kit (PerkinElmer Setus, Branchburg, NJ, USA) for genetic confirmation.

\section{Analysis of Insulin Gene Expression by Northern Blot}

Total RNA was extracted from phosphate-buffered saline (PBS)-washed BM-derived cluster cultures, using RNA-Bee (Tel-Test, Inc. TX, USA) and purified per the manufacturer's guidelines. RNA concentration and purity were determined by routine spectrophotometry. Samples of RNA ( $25 \mu \mathrm{g} /$ lane $)$ were size fractionated on $1 \%$ agarose gels and transferred to nylon membranes (Amersham, Arlington Heights, IL, USA) by capillary action. After crosslinking under UV light, membranes were prehybridizated and then hybridized overnight with specific insulin cDNA that had been labeled with $\left[\alpha^{32} \mathrm{P}\right] \mathrm{dCTP}$ using an Amersham random primer kit. Membrane was subsequently washed under highstringency conditions and exposed to X-omat film (Kodak, NY, USA).

\section{In Situ Hybridization (ISH) with Digoxigenin-Labeled DNA Probes (Boehringer Mannheim)}

Frozen sections of $6 \mu \mathrm{m}$ thickness were fixed for $15 \mathrm{~min}$ in $4 \%$ paraformaldehyde. Rat insulin digoxigenin-labeled DNA probe (Roche, Indianapolis, IN, USA) was denatured at $80^{\circ} \mathrm{C}$ for $5 \mathrm{~min}$ and applied to sections at $52^{\circ} \mathrm{C}$. The sections were coverslipped and sealed with rubber cement for incubation overnight in a hydrated slide box at $52^{\circ} \mathrm{C}$. The following day, the coverslips were carefully removed in preheated 2x SSC buffer, $\mathrm{pH} 7.0$, at $65^{\circ} \mathrm{C}$. The sections were gently washed twice in preheated $50 \%$ formamide in 5x SSC buffer for 5 min each at room temperature and were then gently washed twice in preheated $0.1 \mathrm{x}$ SSC buffer for $5 \mathrm{~min}$ each at $65^{\circ} \mathrm{C}$. Color development was performed at room temperature in buffer (Tris $100 \mathrm{mM}$, NaCl $100 \mathrm{mM}$ and $\mathrm{MgCl}_{2} 50 \mathrm{mM}, \mathrm{pH}$ 9.5) containing NBT and BCIP (Roche). Sections were counterstained with nuclear fast red (Vector Laboratories, Burlingame, CA, USA) and mounted in Cytoseal (Richard-Allan Sci. Kalamazoo, MI, USA). 


\section{Immunocytochemistry}

Immunoactivity was detected using the cytochemical method described by $\mathrm{Oh}$ et al. ${ }^{7}$ Trans-differentiated BM-derived cells were grown on coated coverslips until spheroid colonies formed at approximately 10 days of culture. Individual clusters were retrieved, frozen in OCT at $-70^{\circ} \mathrm{C}$, cut into $6 \mu \mathrm{m}$ sections and placed on Superfrost Plus slides. Sections were then fixed in a $4 \%$ paraformaldehyde/ PBS solution at room temperature for $15 \mathrm{~min}$. The slides were further treated with a $5 \%$ skim milk in TBS-Ca blocking medium for $1 \mathrm{~h}$. These slides were incubated overnight at $4{ }^{\circ} \mathrm{C}$ with one of the following: anti-human Insulin (Dako, Carpinteria, CA, USA), anti-rat insulin (Santa Cruz Biotechnology, Inc., Santa Cruz, CA, USA), anti-human pancreatic polypeptide (Dako), anti-rat somatostatin (Santa Cruz), or anti-rat C-peptide (Linco Research Inc., St Charles, MI, USA) diluted at 1:100. Sections were washed with TBS-Ca, samples were incubated at $4{ }^{\circ} \mathrm{C}$ for $3 \mathrm{~h}$ with secondary antibody: anti-rabbit IgG Texas Red-conjugated, anti-guinea pig IgG Texas Red-conjugated, anti-rabbit IgG Fluoresceine (FITC) conjugated or anti-goat IgG FITC conjugated (Santa Cruz, CA, USA) at a 1:100 dilution with DAPI (Vecter Lab. Burlingame, CA, USA) for nuclear staining. The sections were then photographed using an Olympus microscope and Optronics Digital camera (Olympus, Melville, NY, USA) or a MRC 1024 GS confocal microscope (Bio-Rad, Hercules, CA, USA).

\section{Measurement of Insulin Content and Secretion by Immunoprecipitation-Western Blotting and ELISA}

BM cells were cultured in the presence of 1\% DMSO for 3 days, and changed to DMEM with $4.5 \mathrm{~g} / \mathrm{l}$ glucose and $10 \%$ FBS for 7 days. The medium was then changed to serum-free medium containing $0.5 \% \mathrm{BSA}$ and $5.5 \mathrm{mM}$ glucose to enable the detection of insulin secretion without interference from the fetal serum. The cells were incubated in this condition for $5 \mathrm{~h}$ at $37^{\circ} \mathrm{C}$ followed by washing twice with additional serum-free medium. Highglucose challenge of the cells was achieved by the addition of serum-free media containing $25 \mathrm{mM}$ glucose for a $2 \mathrm{~h}$ at $37^{\circ} \mathrm{C}$. The conditioned media was collected and frozen at $-20^{\circ} \mathrm{C}$ until assayed for insulin content. Intracellular insulin was detected by cell extraction with lysis buffer and a combination of immunoprecipitation and Western blotting, as detailed by Yang et al. ${ }^{16}$ Specifically, the presence of insulin in the culture media and cells lysates was determined by immunoprecipitation with a rabbit polyclonal antiinsulin antibody (Santa Cruz Biotechnology) followed by separation of the precipitated material by SDS-PAGE on $18 \%$ gels, transfer to nylon membranes, and blotting with anti-insulin antibody. A measure of $150 \mu \mathrm{g}$ of cell lysate or $1 \mathrm{ml}$ of culture media was subjected to immunoprecipita- tion. Normal rabbit serum was used as primary antibody control and culture medium containing $0.5 \%$ BSA was used as a control for secreted insulin measurements. Insulin protein was visualized by chemiluminescence. ELISA was performed on the conditioned media to determine insulin secretion using the 1-2-3 Ultra-Sensitive Rat Insulin ELISA kit following the manufacturer's instructions (ALPCO Diagnostics, Windham, NH, USA).

\section{Insulin-Producing Cell Transplantation and Physiological Tests}

Hyperglycemia was induced in 10-week-old male NOD/scid mice (The Jackson Laboratory, Bar Harbor, ME, USA) through intraperitoneal injection of $40 \mathrm{mg} / \mathrm{kg}$ of streptozotocin (STZ) once a day for five consecutive days as described by Yang et al. ${ }^{16}$ Blood glucose level was determined using a standard blood glucose meter (One touch profile, Johnson and Johnson Com., Milpitas, CA, USA). Stable hyperglycemia (blood glucose levels ranging between 300 and $600 \mathrm{mg} / \mathrm{dl}$ ) developed 5-6 days following the last STZ injection.

Under general anesthesia, mice received a renal subcapsular transplant of 150 insulin-producing clusters $(n=9)$, a sham transplant of saline solution $(n=10)$ or 10 million noncultured BM cells $(n=3)$ in the right subcapsular renal space. Blood glucose levels were monitored every 2 days after transplantation for 17 days. Grafts were then removed by unilateral nephrectomy to test for euglycemia reversal and glucose monitoring continued $(n=6)$.

\section{Immuno-gold Labeling and Electron Microscopy}

Insulin-producing cell clusters were fixed in $0.1 \%$ glutaraldehyde $/ 2 \%$ formaldehyde in $0.1 \mathrm{M}$ cacodylate buffer, transferred to $0.1 \mathrm{M}$ cacodylate buffer, and embedded. Ultrathin sections were sequentially treated with $10 \% \mathrm{H}_{2} \mathrm{O}_{2}$, washed with $0.9 \% \mathrm{NaCl}$, blocked with $3 \% \mathrm{BSA}$, incubated overnight with the primary antibody in PBS supplemented with $0.5 \%$ BSA (rabbit anti-rat insulin, 1:100, Santa Cruz), washed with PBS, and reblocked with goat serum. Binding of the primary antibody was visualized with gold-labeled secondary antibody (15-nm gold particles, goat-anti-rabbit, Amersham Pharmacia, Piscataway, NJ, USA), followed by counterstaining of the sections for electron microscopy.

\section{Results}

BM-Derived Cells Trans-Differentiation into Endocrine Cells

BM cells were plated on $0.3 \%$ rat tail collagen (RTC) coated coverslips contained within six-well culture dishes. BM cells were cultured with $1 \%$ dimethyl sulfoxide (DMSO) in serum-free DMEM for 3 days 
and then maintained for an additional 7 days in a DMSO-free DMEM medium containing 10\% FBS with either a low $(5.5 \mathrm{mM})$ or high $(25 \mathrm{mM})$ concentration of glucose. Cells cultured under high-glucose conditions began to form small spheroid clusters at approximately day 7 (Figure 1a). Following day 10, under the same conditions, the number and dimension of these spheroid cell clusters were markedly increased (Figure $1 \mathrm{~b}$ and c). The three-dimensional cell growth morphology closely resembled islet-like clusters, as previously described by other investigators. ${ }^{13,15,16}$ The results of the experimental approach using either low- or high-glucose medium in inducing spheroid formation are summarized in Figure 1d. The number of clusters formed in low-glucose culture conditions on a $22 \times 22 \mathrm{~mm}^{2}$ coverslip was $17 \pm 11$.3. However, under high-glucose condition the number of clusters obtained were $157 \pm 32.9$ (Figure 1d). Also, it should be noted that the cluster size under low-glucose conditions was considerably smaller as compared to that of high-glucose condition (data not shown). Based on the culture conditions and the harshness of the first 3 days, we looked at viability of the BM cells. At time zero, 2-h postculture the viability of these cells was at $95 \%$ by Trypan blue-exclusion technique. The cells at day 3 of culture (1\% DMSOserum free) revealed that the cells were $97.6 \%$ viable by Trypan blue-exclusion and propidium iodide (PI) FACS analysis. Lastly, we examined the clusters formed in culture (day 10) for cell viability. The clusters were disassociated and examined by Trypan blue-exclusion at PI FACS. The assays revealed very little cell death within the formed clusters with cell viability in the $99 \%$ range. Thus, the harshness of the culture conditions ( 3 days in serum-free $1 \%$ DMSO media) did appear to select for a subpopulation of BM cells, which, when switched to a high-glucose serum-supplemented media, were able to proliferate and form clusters.

\section{Gene Expression of Endocrine Cell Markers by the BM-Derived Clusters}

To determine whether the islet-like clusters appearing in the BM-derived cell cultures may have transdifferentiated into endocrine hormone-producing
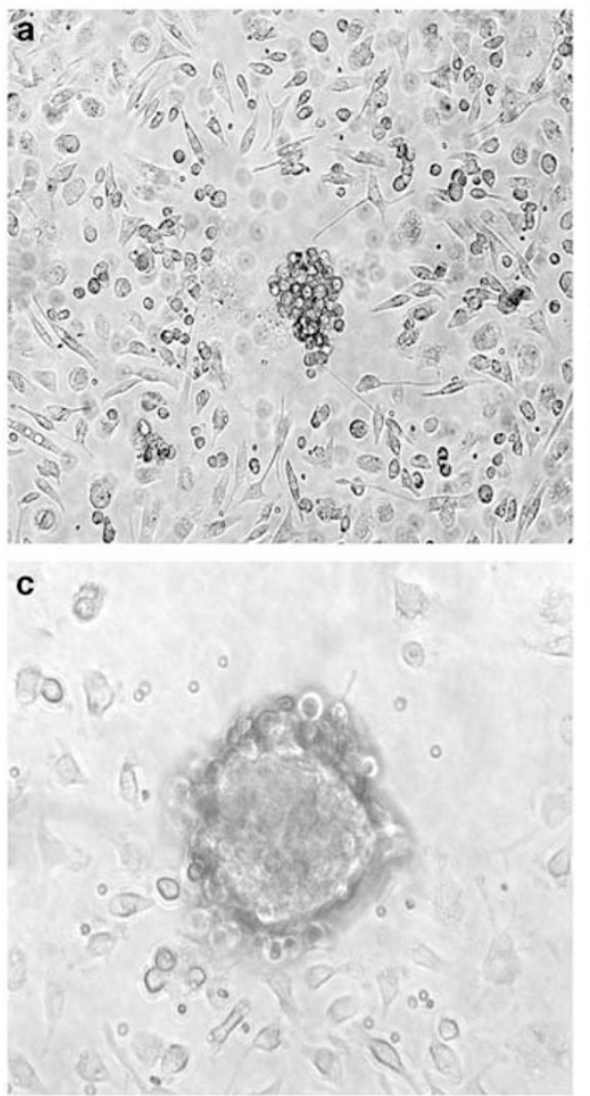

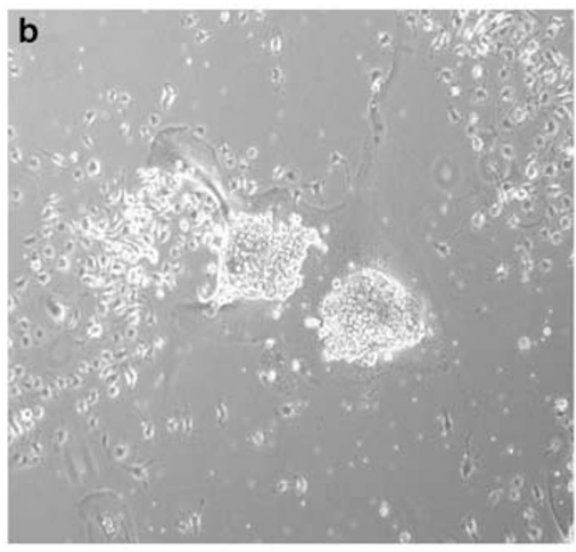

d

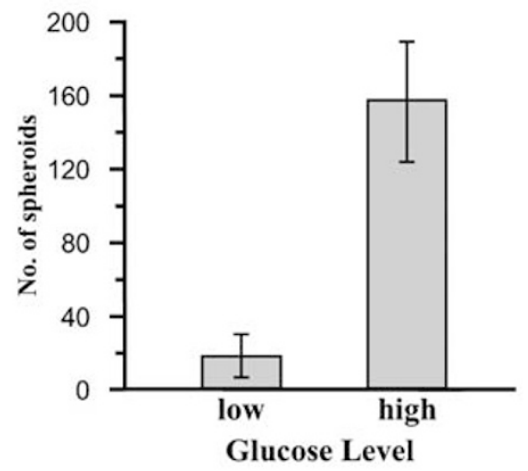

Figure 1 BM cells differentiated into pancreatic-like cells via in vitro culture system. Trans-differentiation of BM cells under conditions, which induce an endocrine cell phenotype: BM cells cultured in the serum-free medium for 3 days in $1 \%$ DMSO then cultured in the $10 \%$ FBS and high glucose $(25 \mathrm{mM})$ for an additional 4 days. Small clusters begin to form and continued to expand forming a tightly organized mass of cells (a). Multiple clusters can be seen in single fields (b), and at higher magnifications they appear to have defined edges and structure (c). Low-glucose conditions produce 17.3 \pm 11.3 clusters while high-glucose conditions for 10 days gave a mean value of $157.5 \pm 32.9$ clusters $\left(n=8 / 22 \times 22 \mathrm{~mm}^{2}\right.$ coverslips, three separate experiments) clusters (d). Original magnification for (a) and $(\mathbf{c}): \times 40$, with magnification for $(\mathbf{b}): \times 20$. 
cells, the expression of endocrine cell genes was examined by RT-PCR. In order to ascertain whether pancreatic lineages are originally present in the bone marrow, we tested total RNA extracted from freshly isolated BM cells by RT-PCR for gene expression of pancreatic specific transcription factors and the Glut-2 and endocrine genes. Transcription factors, Glut-2 and endocrine pancreatic mRNAs were not detected in the freshly isolated BM cells at day 0 (Figure 2a and b, D0). However, when BM cells were cultured with 1\% DMSO for 3 days, PDX-1, NKX2.2 and NKX6.1 messages were strongly expressed (Figure 2a, D3). Furthermore, the Glut-2 gene was not expressed in D0 and D3, but the message was expressed following the change to high-glucose culture medium (Figure 2a days 7 and 10). Endocrine messages demonstrate that expression of genes occurred in BM cell cultures treated with 1\% DMSO. Insulin (I and II), glucagon, somatostatin and pancreatic polypeptide mRNAs were detected during the initial 3 days of culture (Figure 2b, D3). Further investigation of cultures in high-glucose DMEM with 10\% FBS also showed the expression of these mRNAs at days 7 and 10 (Figure 2b). These PCR products were further analyzed by sequencing and compared to GenBank for confirmation (data not shown).

a

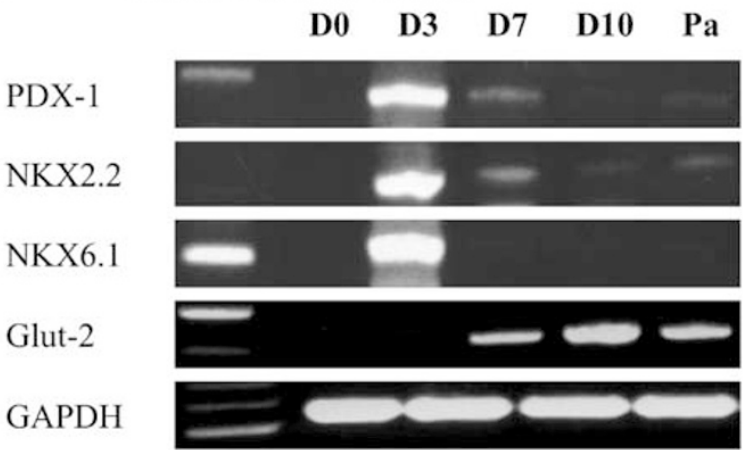

b

\section{Endocrine Genes}

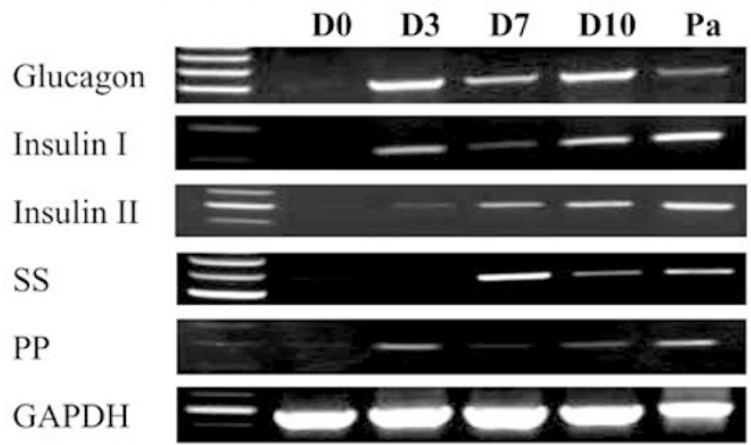

Figure 2 Pancreatic gene expression in noncultured and cultured $\mathrm{BM}$ derived cells. RT-PCR analysis for expression of transcription factors, Glut-2 (a) and pancreatic endocrine genes (b) in adult pancreas (control), noncultured BM-derived cells and cultured BM-derived cells.
In addition, we also examined the mRNA expression for insulin by Northern blot and in situ hybridization of BM-derived clusters. Figure 3a shows the presence of insulin mRNA in cultured BM-derived insulin-producing clusters and INS-1 cells (positive control), but fresh BM cells did not express insulin message. Expression of the insulin gene was also examined by ISH of the BM-derived clusters. Figure $3 \mathrm{~b}$ represents ISH for insulin on noncultured BM cells. As seen in the figure the noncultured BM cells are negative for insulin expression. While Figure 3c indicates the presence of the insulin expression in INS-1 cells (positive control). Figure $3 \mathrm{~d}$ and e show the presence of
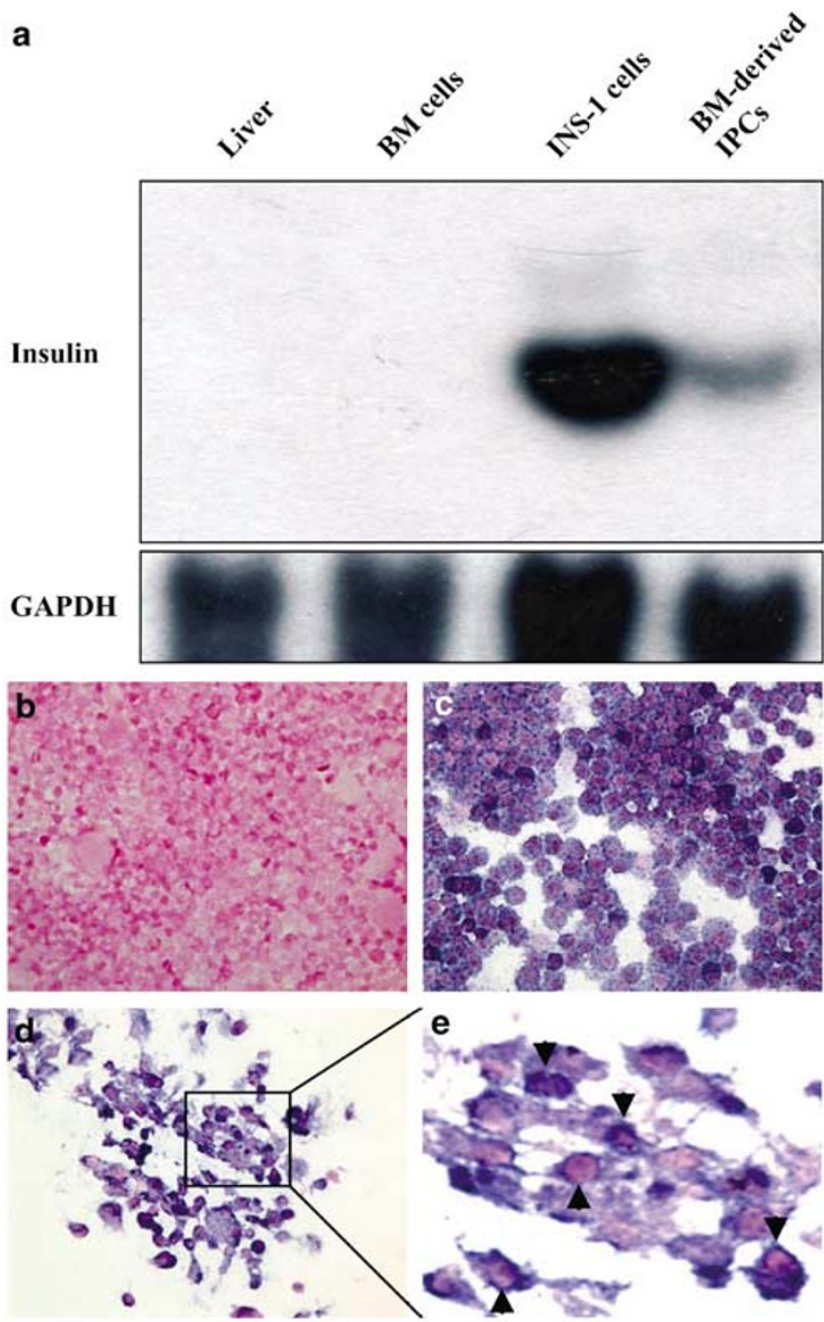

Figure 3 Northern blot and ISH analyses of expression of insulin message in cultured BM cells. (a) Insulin mRNA levels in BM derived cells: Northern analysis for expression of insulin mRNA in normal liver (negative control), INS-1 cell (positive control), cultured BM derived cells and noncultured BM-derived cells. (be) ISH of insulin mRNA in whole BM cells (b; Negative control), INS-1 cell (c; Positive control) and frozen sections of clusters (d and e). ISH analysis at low and high magnifications (d and e). The cells are positive for insulin mRNA expression (blue) and counter stained with nuclear fast red. (b-d) photomicrographs were taken using $\times 60$ and $\times 100$ oil immersion objectives, respectively. 
insulin mRNA in the cytoplasm of the BM-derived clusters using a DIG-labeled insulin-oligonucleotide probe. Insulin mRNA is clearly visible in a majority of the cells within the BM-derived clusters. These results indicate that BM-derived clusters may possess the potential to trans-differentiate into cells found within the islets of Langerhans.

\section{Protein Analysis of BM-Derived Insulin-Producing Cells}

To investigate the expression of pancreatic hormones, immunofluorescence analyses was performed for insulin, somatostatin and pancreatic polypeptide in BM-derived clusters. BM cells cultured with DMSO and under high-glucose condition were capable of cluster formation. The islets of Langerhans in normal pancreas were shown to strongly express insulin (Figure 4a). Furthermore, other cells found in islets of Langerhans express somatostatin (Figure $4 \mathrm{~b}$ ) and pancreatic polypeptide (Figure 4c). Figure 4d represents noncultured BM stained for insulin. In addition, immunofluorescence was performed for somatostatin and pancreatic polypeptide revealing no positive staining (data not shown). However, the BM derived insulinproducing clusters did express these cytoplasmic proteins (Figure $4 \mathrm{e}-\mathrm{g}$ ). In these BM-derived cells, a higher number of insulin-positive cells were observed, corresponding to approximately $80 \%$ of the total cell population (Figure 4e). As expected, the number of insulin-positive cells observed was significantly higher than the number of cells expressing either somatostatin (Figure 4f) or pancreatic polypeptide (Figure 4g).

The trans-differentiated BM-derived cells, or insulin-producing clusters, synthesize and store detectable amounts of insulin during glucose challenge as determined by immunoprecipitation and Western blot analysis (Figure 5a). It was found that during glucose challenge, the cell lysate of the insulin-producing cell clusters contained proinsulin, while the media revealed the two-chain form of insulin. In addition to the Western analysis, we performed an ELISA assay to quantify the insulin being released into the media. In two separate experiments, BM-derived insulin-producing cell clusters (approx. 90 clusters per experiment) were challenged with $25 \mathrm{mM}$ glucose for $2 \mathrm{~h}$ which resulted in secretion of roughly $186 \mathrm{ng} / \mathrm{ml}$ of insulin into the media (Figure 5b). However, cells cultured in a low-glucose media $(5.5 \mathrm{mM})$ as well as media alone, showed no insulin reactivity (Figure $5 \mathrm{~b}$ ).

In addition, we also assessed the C-peptide expression of the BM-derived insulin producing clusters. Cells positive for C-peptide staining were seen throughout the clusters (Figure 6c-e). A comparison of the C-peptide staining pattern of a positive control cell line, INS-1, which overexpresses insulin (Figure 6a and b), to our insulin-
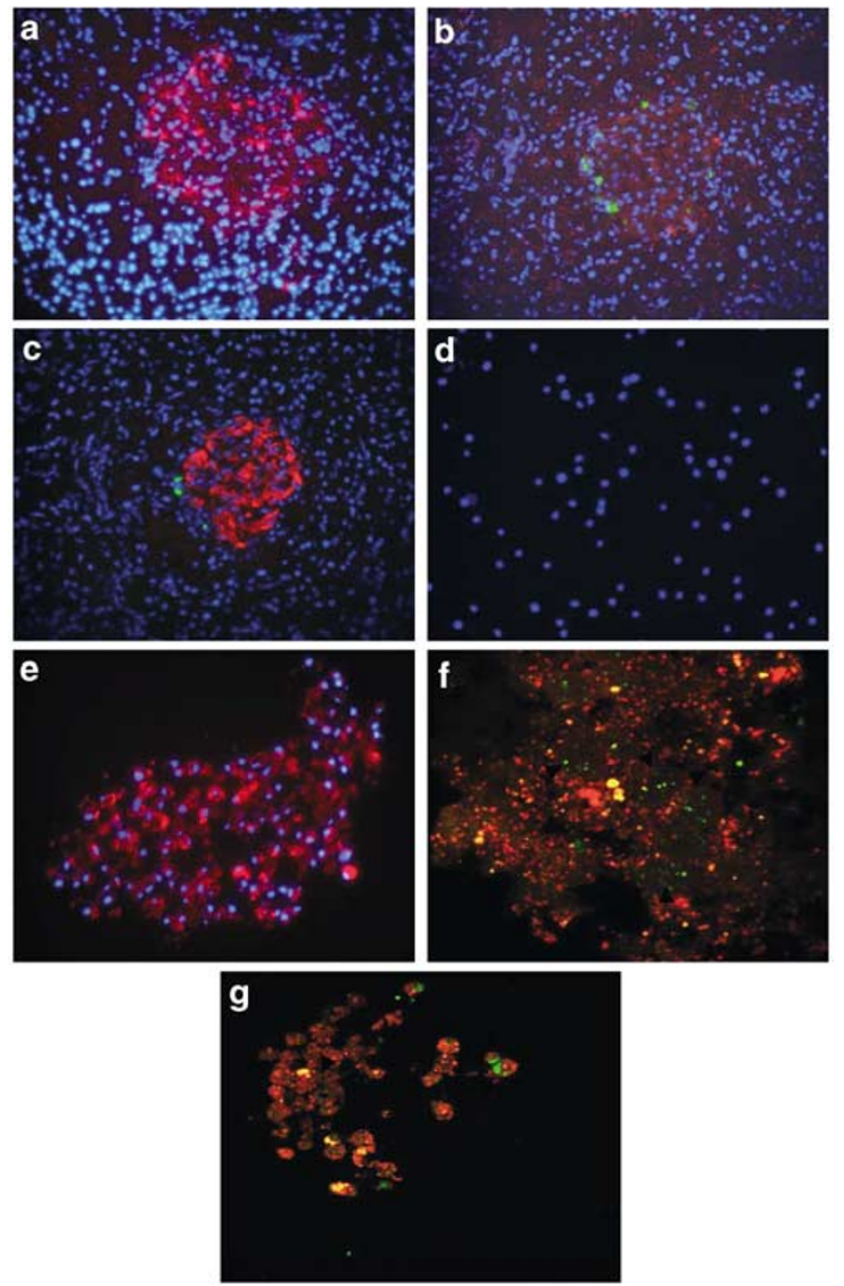

Figure 4 Immunofluorescence reveals BM-derived cells differentiated into pancreatic-like in vitro. Immunohistochemistry for insulin (a; Texas red) and double staining of insulin (Texas Red) with somatostatin (b; FITC) or pancreatic polypeptide (c; FITC) on normal pancreas, and nonculture BM cells counterstained using DAPI (blue) for nuclear staining; no immunostaining is observed (d). Immunofluorescence staining for insulin (Texas Red) on whole mount in BM derived cells, day 10 and frozen section of isolated cluster at $\times 40$ : insulin immunoreactivity is present in the spheroid (e). Confocal photomicrographs of dual immunofluorescence for insulin (Texas Red) with somatostatin (FITC) or pancreatic polypeptide (FITC) on frozen sections of BM-derived cells, (f and $\mathbf{g}$ respectively). Original magnification is (b and $\mathbf{d}$ ), $\times 40$ and $(\mathbf{c}), \times 20$ objective. Photomicrographs for (f) and (g) were taken on MRC 1024 GS confocal microscope using a $\times 60$ oil immersion objective (f), and a $\times 40$ (g) objective.

producing cells, showed a pattern of staining similar in nature. We also examined this immunohistostainning using deconvolution microscopy (Delta Vision, Applied Precision Inc. WA, USA) to pinpoint the Cpeptide staining within the cytoplasm of our insulin-producing cells (Figure 6e). These results suggest that BM-derived clusters may possess the function of insulin secretion during glucose challenge, as well as displaying morphology similar to Langerhans islets. 

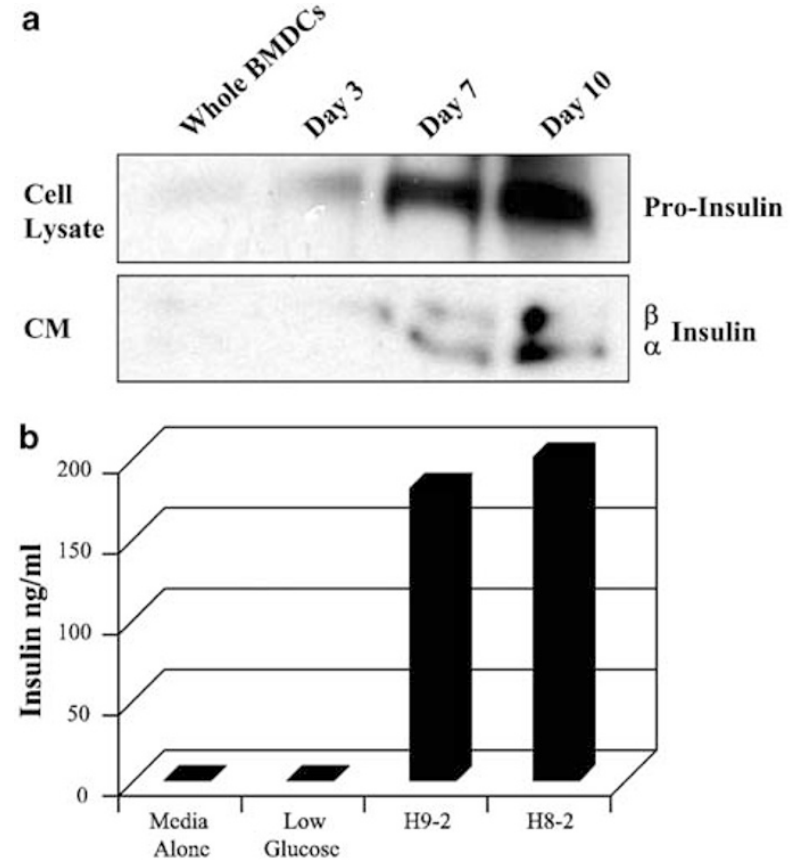

Figure 5 Analysis of secretion of insulin following glucose challenge in BM derived clusters cultures. (a) Western-blot analysis of insulin secretions measured following collection of cell lysates and culture-conditioned media. (b) ELISA assay for insulin in conditioned media from BM-derived cells exposed to low- $(5.5 \mathrm{mM})$ or high- $(25 \mathrm{mM})$ glucose conditions for $2 \mathrm{~h}$. After exposure to high glucose both H9-2 (83 clusters) and H8-2 (94 clusters) showed secretion of insulin into the media (177.8 and $196.0 \mathrm{ng} / \mathrm{ml}$, respectively). Whereas, the BM-derived cells exposed to low-glucose conditions and media alone showed no reactivity to insulin in the assay.

\section{Rescue and Survival of Chemically Induced Diabetic Mice Following Insulin-Producing Cell Transplantation}

Previous studies have shown that maturation to functional islet cells in vitro requires implantation into an in vivo environment. ${ }^{15}$ The ability of insulinproducing cells to reverse hyperglycemia was examined in vivo using a STZ-induced diabetes NOD/scid mouse model. Multiple treatments of STZ chemically induced a diabetic state in NOD/scid mice. Nine diabetic mice received roughly $150 \mathrm{BM}-$ derived insulin-producing clusters transplanted into the renal subcapsular space. The mice receiving the transplant began to normalize their blood glucose levels within 2-3 days (Figure 7). Approximately 17 days post-transplantation, six of the nine mice underwent a nephrectomy to remove the graft. As seen in the graph, these animals became hyperglycemic and died shortly thereafter. The animals that retained the transplanted insulinproducing clusters maintained fairly normal glucose levels for the remainder of the study. Our results suggest that the clusters hold the potential to mature into fully functioning-endocrine cells similar to the islets of the pancreas. Control animals that did not receive implants or received noncultured BM cells
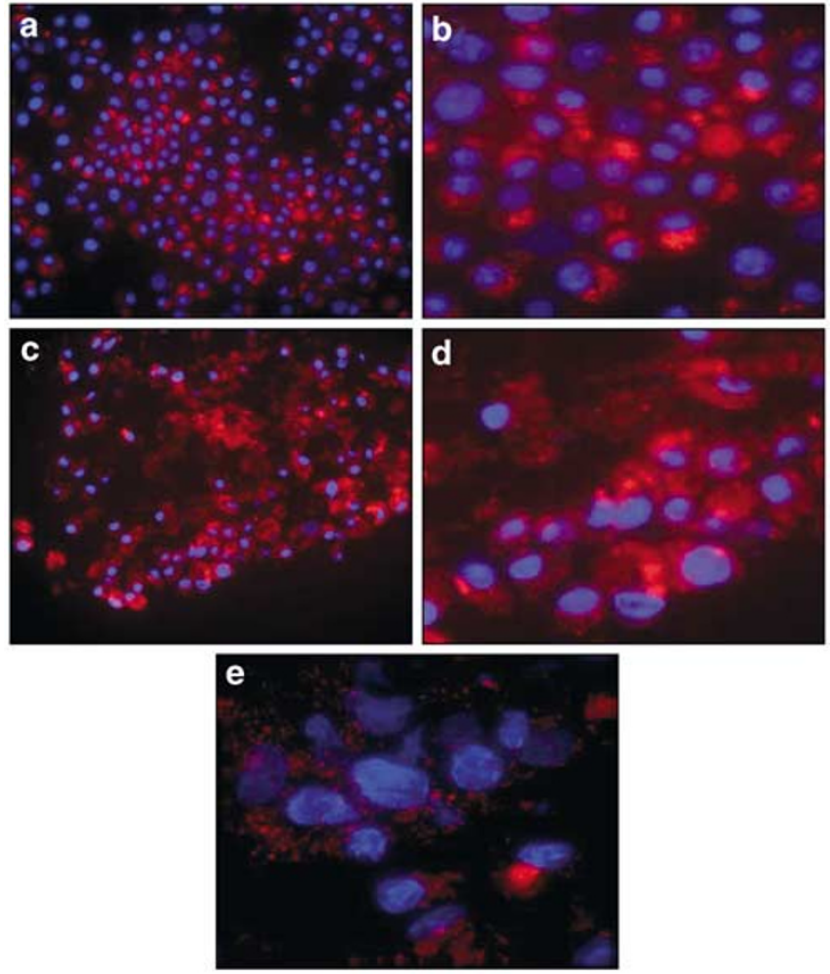

Figure 6 C-peptide analysis of BM-derived cells. Frozen sections of BM-derived cells were immunostained in order to determine the presence of processed proinsulin, which is C-peptide. INS-1 cells (a and $\mathbf{b}$, positive control), BM-derived cells (c, $\mathbf{d}$ and $\mathbf{e}$ ) were immunostained for C-peptide. As seen in the high-magnification photomicrograph (d), the cells within the cluster show distinct staining similar to the INS-1 positive control cells (b). Deconvolution microscopy was used to pinpoint the C-peptide staining within the cytoplasm of our insulin-producing cells (e). Original magnifications are as follows: (a) and (c): $\times 40$ and (b) and (d): $\times 60,(\mathbf{e}) \times 100$ oil immersion.

exhibited persistent hyperglycemia followed by death.

The kidneys that received transplants were excised and further analyzed by conventional hematoxylin/eosin staining, immunohistochemistry, and ISH for insulin expression (Figure 8). Histology of the kidney capsules from animals that received a transplant of insulin-producing clusters was examined (Figure 8b). As seen in the photomicrograph the transplanted clusters were able to engraft under the capsule. The transplanted clusters were positive for insulin by immunohistochemistry (Figure 8c), and insulin gene expression was confirmed by ISH (Figure 8d). Normal kidney tissue, which did not receive a cluster transplant did not hybridize with the insulin probe demonstrating the specificity of the probe for insulin mRNA (Figure 8e). These results show that insulin-producing clusters retain their ability to secrete insulin in vivo and respond to the high-blood glucose levels in the diabetic mice. 


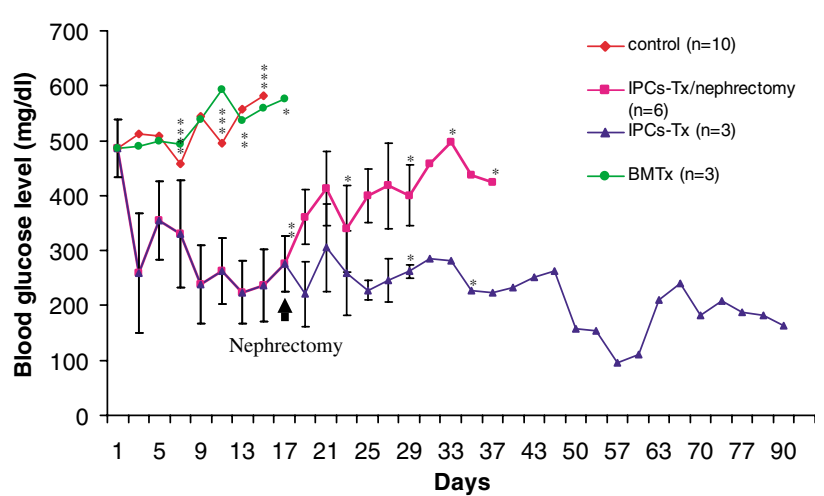

Figure 7 Transplantation of insulin-producing cells into STZtreated diabetic NOD/scid mice. To ascertain the functionality of these insulin-producing clusters and determine the changes of blood glucose levels over time, insulin-producing cells were transplanted into chemically induced diabetic mice. Shown in the graph are control STZ-treated mice transplanted with saline $(n=10)$ or noncultured BM-cells $(n=3)$, and mice transplanted with approximately $150 \mathrm{BM}$-derived insulin-producing clusters $(n=9)$. In order to determine whether the transplanted clusters were responsible for the normalization of glucose levels, the insulin-producing cell transplanted mice were separated into two groups. One group was allowed to maintain the transplanted graft (non-nephrectomy; $n=3$ ), while the second group of transplanted mice underwent a nephrectomy (graft/nephrectomy; $n=6$ ). As seen in the graph, those mice that had the graft removed became hyperglycemic and eventually died (*; end point of death).

\section{Ultra-Structural Analysis of Insulin Producing Cell Clusters}

Ultra-structural analysis of the insulin-producing clusters was also performed. An electron-micrograph of BM-derived trans-differentiated cells at a low magnification reveals structures typical of a secretory cell, including rough endoplasmic reticulum, Golgi complex, a few large vacuoles and secretory vesicles containing dense granules (Figure 9a). In addition, microvilli are visible on their surface, which has been described as a characteristic of $\beta$-cells. ${ }^{20}$ Also, many granules seen in the insulinproducing cell clusters exhibited a pale nature with crystalline-like features (Figure 9b). Lastly, immunogold electron microscopy revealed insulin within the small secretory vesicles of the insulin-producing clusters (Figure 9c-g). Gold-labeling detected faint globular structures of differing size filled with floccular, low-density material (Figure 9c) as well as granules with an electron-dense core at the apical pole of the cells (Figure $9 \mathrm{~d}-\mathrm{g}$ ). The immunogold labeling of the insulin-producing clusters appears to resemble that of normal pancreatic $\beta$-cell, as previously described. ${ }^{21}$ Figure $9 \mathrm{~h}$ and $\mathrm{i}$ represents positive and negative controls for immnunogold labeling, respectively. Figure 9h shows INS-1 cells, which is an over expressing cell line, with several positive particles per secretory granule. The INS-1 cells were presented only to validate the assay and not to correlate insulin-positive signal between the INS-1 cells and the BM-derived insulin-producing clusters. The size and location of the gold labeling of

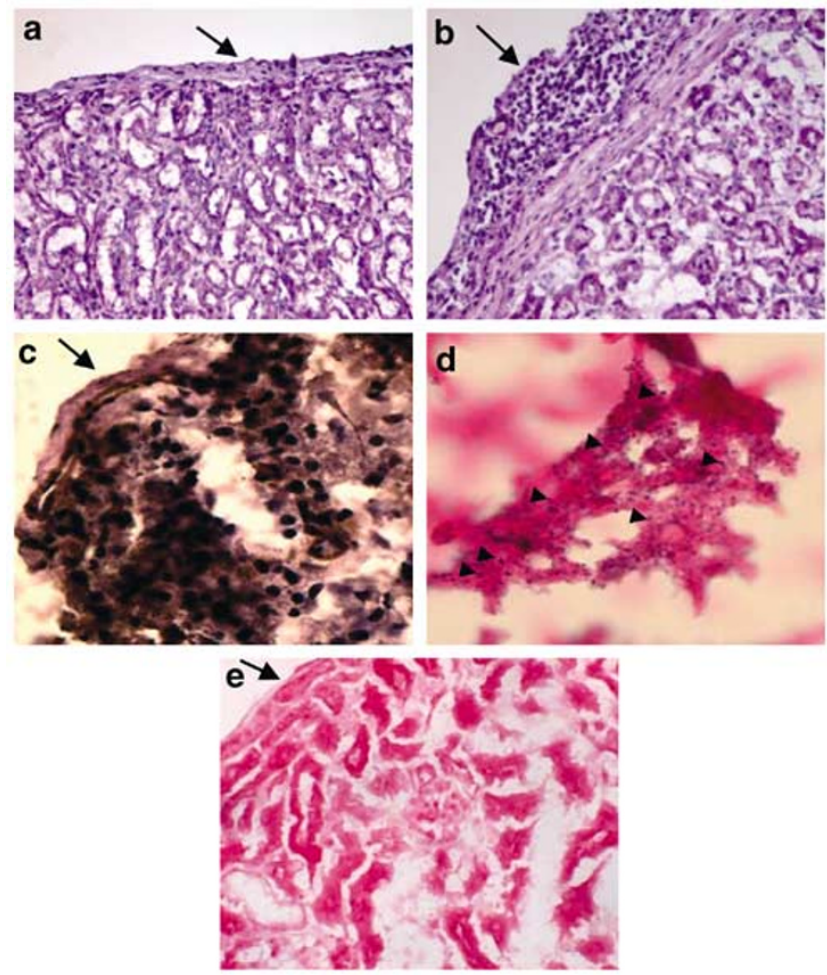

Figure 8 Analysis of graft following kidney excision via immunohistochemistry and ISH for insulin expression. $\mathrm{H}$ and $\mathrm{E}$ staining of engrafted cells under the kidney capsule reveals that the cells have become cuboidal which is typical of the mature islet cell structure seen in adult pancreas (b) and nontransplanted animal kidney (normal control, a). Insulin staining illustrates that most of the grafted cells are still positive (c). With ISH the grafted cells are positive for expression of the insulin mRNA (d). Nontransplanted kidney tissue as a negative control for ISH (e). (c) and (d) were prepared as serial sections of kidney tissue from BM-derived insulin-producing clusters transplanted animals. The large arrows in (a), (b), (c) and (e) represent the kidney capsule, while the arrowheads indicated positive signals for insulin (d). Original magnification is $\times 60$.

the INS-1 cells closely resembles our insulinproducing clusters. Nonspecific labeling was absent in the nucleus and elsewhere throughout the cell (Figure 9i). The ultrastructural features typical of an adult $\beta$-cell found within the insulin-producing clusters suggests these cells may have truly transdifferentiated during culture into $\beta$-like cells capable of producing insulin.

\section{Discussion}

The data presented demonstrates that adult BM cells appear to trans-differentiate into functional endocrine cells, capable of producing and secreting insulin under high-glucose culture condition. When insulin-producing clusters are transplanted into STZ treated NOD/scid mice, blood glucose levels were normalized. Previously, several reports have demonstrated the presence of progenitor cells within pancreatic islets that are capable of differentiating 

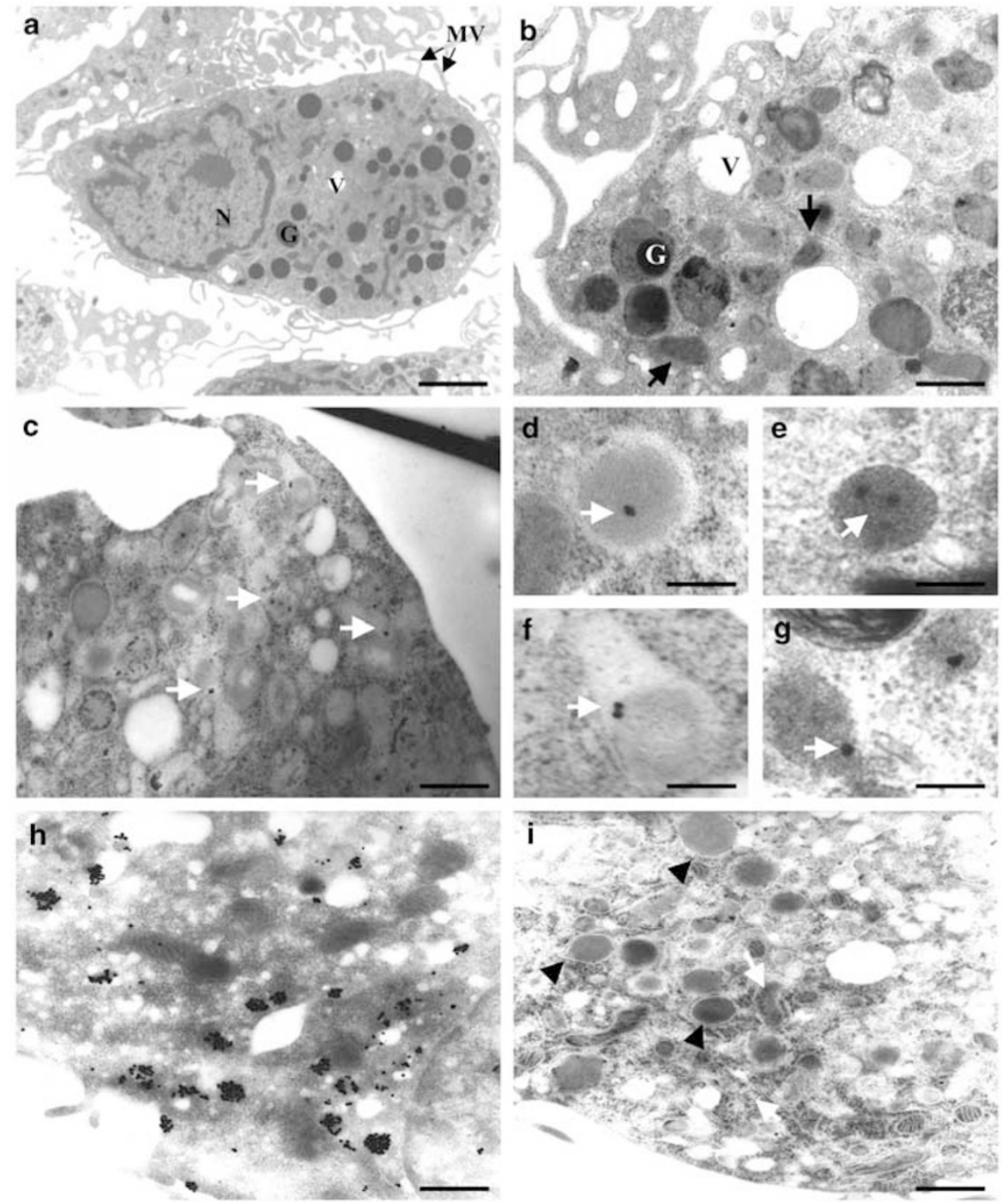

Figure 9 Electron microscopy and immunoelectron microscopy of BM-derived insulin-producing cell spheroids. Electron microscopy of day 10 insulin-producing cells characterizes their ultrastructure. Secretory granules are densely packed within the cytoplasm of the trans-differentiated cell. Along the cell membrane, microvilli can be seen (a). This has been reported to be another characteristic of mature $\beta$-cells. At higher magnification, secretory granules are seen throughout the cytoplasm of the cell (b). The black arrow in (b) indicates crystalline formation within the granular cores. Continued characterization of the trans-differentiated cells through immunogold staining was performed. Postembedding immunogold staining for insulin is shown in (c-g), positive control for immunogold labeling (h) and negative control (i). The positive control is only a representative electron-photomicrograph for the immunogold assay and is not showing a correlation of insulin expression between INS-1 cells and the insulin-producing clusters. Black arrowheads shown in (i) indicate secretory granules illustrating no evidence of insulin expression by the immunogold staining. White arrows shown in $(\mathbf{c}-\mathbf{g})$ indicate immunogold particles concentrated in the secretory granules of the trans-differentiated cells. Scale bar, $5 \mu \mathrm{m}$ (a, h and $\mathbf{i}), 1 \mu \mathrm{m}$ (b and $\mathbf{c}$ ) and $0.2 \mu \mathrm{m}(\mathbf{d})-(\mathbf{g})$. N, nucleus; V, vacuole; G, granule; MV, microvilli.

into insulin-producing cells. ${ }^{15,22}$ Other reports have stated that hepatic oval cells, embryonic stem cells and splenocyte are capable of differentiating into cells with a pancreatic endocrine phenotype. ${ }^{16-18,23}$ The present report indicates an additional source of cells capable of differentiating into pancreatic endocrine cells both phenotypically and functionally.

Hematopoietic stem cells (HSC), are normally present in adult marrow and have been shown to differentiate into a variety of cell types, including bone, muscle, fat, cartilage, cardiomyocytes, astro- cytes and hepatic cells. ${ }^{5,24-29}$ Recently, two reports have described the pluripotency of a mesenchymal cell also known as multipotent adult progenitor cells (MAPC). When derived from adult BM, MAPCs can be kept in culture for an extended period of time and can also differentiate into both hepatic and neural lineages. ${ }^{30,31}$ Although HSCs and MAPCs can differentiate into several lineages, to date no pancreatic cells have been described. A recent paper by Lumelsky et $a l^{17}$ demonstrated that embryonic stem cells may possess the ability to become $\beta$-like insulin-producing cells. However, 
a paper published by Rajagopal et $a 1^{32}$ observed that these embryonic ' $\beta$-like' cells did not produce insulin, but merely took up insulin from the media and subsequently appeared positive for insulin via immunohistochemistry. This finding suggested that several techniques over and above RT-PCR and immunohistochemistry would be necessary to confirm true trans-differentiation of a cell into an insulin-secreting $\beta$-cell. These suggestions including the use of both Northern and Western analyses, electron microscopy and long-term animal studies have become the new criteria which investigators must now meet in order to claim that cells have trans-differentiated into functioning $\beta$-cells.

Our data demonstrate that adult bone marrow cells can trans-differentiate into functional endocrine cells capable of the production and secretion of physiologically active insulin when cultured under DMSO and high-glucose conditions. The paradigm of BM cell 'trans-differentiation' into multiple cell lineages has been challenged by the observation of cell fusion in vitro of pluripotent cells with differentiated cells thus adapting a 'differentiated' phenotype. ${ }^{33,34}$ More recently, two reports have shown that the fusion phenomenon appears to play an important role in a certain type of liver regeneration. ${ }^{35,36}$ These two reports indicate cell fusion as the major mechanism in the regeneration process of the $\mathrm{Fah}^{-/-}$liver. $^{35,36}$ However, Ianus et $a l^{37}$ recently reported that $\mathrm{BM}$ cells could differentiate into $\beta$-cells in vivo using an elegant Lox/Cre-GFP system demonstrating fusion was not a mechanism in $\beta$-cell regeneration. These papers raise several questions as to the role that the type and model of injury may play in dictating the specific processes involved in regenerating the injured organ.

In contrast to what has previously been reported, we have shown that the manipulation of BM cells toward being capable of secreting insulin may be accomplished with relative ease. The trans-differentiation method as presented, only requires 10 days from BM-derived cells to insulin-producing clusters rather than months, thus providing an accessible cell source and a simple method for the cellular treatment of diabetes. In addition, our results illustrate that BM cells can trans-differentiate into a pancreatic lineage in vitro without fusion and can function as insulin producing cells both in vivo and in vitro. It may also be clinically relevant that these cells may not present themselves as fully mature $\beta$-cells thereby evading the cycle of $\beta$-cell destruction by the immune system. However, further characterization of these cells will be required to determine if this is indeed the case.

In conclusion, our findings present an evidence that BM may include a pancreatic progenitor cell capable of differentiating into functioning endocrine hormone-producing cells. The differentiated cells possess storage granules and produce proinsulin which is cleaved into active insulin in response to glucose challenge. When transplanted into diabetic mice, the cells retain their properties of storage, processing and regulated secretion of insulin to normalize blood glucose levels. Despite our ability to induce the secretion of detectable levels of insulin in vitro in the presence of high glucose by BMderived insulin-producing clusters, it will be essential to understand what mechanisms are involved during the trans-differentiation process allowing $\mathrm{BM}$ cells to mature and differentiate into fully functional $\beta$-like cells. Our results suggest that BM cells may provide researchers a powerful tool in the study of pancreatic islet development and function, as well as offering a new potential instrument for the treatment of diabetes.

\section{Acknowledgements}

We wish to thank Dr Jill Verlander, Dr Sharon W Matthews, Ms Debbie Akin, Ms Maureen A Petersen, and Ms Stephanie Mauthner for their excellent expertise and assistance in performing electron microscopy and printing the electron micrographs used in Figure 9. We also wish to thank Drs Mark Atkinson and Brant Burkhardt in providing the insulin ELISA results used in Figure 5b, and Dr Sally Litherland for her help in the deconvolution photomicrograph in Figure 6e. National Institute of Health Grants DK60015 and DK58614 awarded to BEP funded this research.

\section{References}

1 Weissman IL. Translating stem and progenitor cell biology to the clinic: barriers and opportunities. Science 2000;287:1442-1446.

2 Steindler DA, Pincus DW. Stem cells and neuropoiesis in the adult human brain. Lancet 2002;359:1047-1054.

3 Scott EW, Simon MC, Anastasi J, et al. Requirement of transcription factor PU.1 in the development of multiple hematopoietic lineages. Science 1994;265: 1573-1577.

4 Petersen BE, Zajac VF, Michalopoulos GK. Hepatic oval cell activation in response to injury following chemically induced periportal or pericentral damage in rats. Hepatology 1998;27:1030-1038.

5 Petersen BE, Bowen WC, Patrene KD, et al. Bone marrow as a potential source of hepatic oval cells. Science 1999;284:1168-1170.

6 Theise ND, Badve S, Saxena R, et al. Derivation of hepatocytes from bone marrow cells in mice after radiation-induced myeloablation. Hepatology 2000;31: 235-240.

7 Oh SH, Miyazaki M, Kouchi H, et al. Hepatocyte growth factor induces differentiation of adult rat bone marrow cells into a hepatocyte lineage in vitro. Biochem Biophys Res Commun 2000;279:500-504.

8 Hellerstrom C. The life story of the pancreatic $\beta$-cell. Diabetologia 1984;26:393-400.

9 Rosenberg L, Vinik AI. Trophic stimulation of the ductular-islet cell axis: a new approach to the 
treatment of diabetes. Adv Exp Med Biol 1992;321: 95-104.

10 Swenne I. Pancreatic beta-cell growth and diabetes mellitus. Diabetologia 1992;35:193-201.

11 Guz Y, Nasir I, Teitelman G. Regeneration of pancreatic beta cells from intra-islet precursor cells in an experimental model of diabetes. Endocrinology 2001; 142:4956-4968.

12 Shapiro AM, Lakey JR, Ryan EA, et al. Islet transplantation in seven patients with type 1 diabetes mellitus using a glucocorticoid-free immunosuppressive regimen. N Engl J Med 2000;343:230-238.

13 Bonner-Weir S, Taneja $\mathrm{M}$, Weir GC, et al. In vitro cultivation of human islets from expanded ductal tissue. Proc Natl Acad Sci USA 2000;97:7999-8004.

14 Halvorsen T, Levine F. Diabetes mellitus-cell transplantation and gene therapy approaches. Curr Mol Med 2001;1:273-286.

15 Ramiya VK, Maraist M, Arfors KE, et al. Reversal of insulin-dependent diabetes using islets generated in vitro from pancreatic stem cells. Nat Med 2000;63: 278-282.

16 Yang L, Li S, Hatch $\mathrm{H}$, et al. In vitro trans-differentiation of adult hepatic stem cells into pancreatic endocrine hormone-producing cells. Proc Natl Acad Sci USA 2002;99:8078-8083.

17 Lumelsky N, Blondel O, Laeng P, et al. Differentiation of embryonic stem cells to insulin-secreting structures similar to pancreatic islets. Science 2001;292:13891394.

18 Soria B, Roche E, Berna G, et al. Insulin-secreting cells derived from embryonic stem cells normalize glycemia in streptozotocin-induced diabetic mice. Diabetes 2000;49:157-162.

19 Michalopoulos G, Pitot HC. Primary culture of parenchymal liver cells on collagen membranes. Morphological and biochemical observations. Exp Cell Res 1975;94:70-78.

20 Orci L, Thorens B, Ravazzola M, et al. Localization of the pancreatic beta cell glucose transporter to specific plasma membrane domains. Science 1989;245: 295-297.

21 Bendayan M. Ultrastructural localization of insulin and C-peptide antigenic sites in rat pancreatic B cell obtained by applying the quantitative high-resolution protein A-gold approach. Am J Anat 1997;185: 205-216.

22 Fernandes A, King LC, Guz Y, et al. Differentiation of new insulin-producing cells is induced by injury in adult pancreatic islets. Endocrinology 1997;138: 1750-1762.
23 Kodama S, Kuhtreiber W, Fujimura S, et al. Islet regeneration during the reversal of autoimmune diabetes in NOD mice. Science 2003;302: 1223-1227.

24 Rickard DJ, Sullivan TA, Shenker BJ, et al. Induction of rapid osteoblast differentiation in rat bone marrow stromal cell cultures by dexamethasone and BMP-2. Dev Biol 1994;161:218-228.

25 Ferrari G, Cusella-De Angelis G, Coletta M, et al. Muscle regeneration by bone marrow-derived myogenic progenitors. Science 1998;279:1528-1530.

26 Umezawa A, Maruyama T, Segawa K, et al. Multipotent marrow stromal cell line is able to induce hematopoiesis in vivo. J Cell Physiol 1992;151 197-205.

27 Ashton BA, Allen TD, Howlett CR, et al. Formation of bone and cartilage by marrow stromal cells in diffusion chambers in vivo. Clin Orthop 1980;151: 294-307.

28 Makino S, Fukuda K, Miyoshi S, et al. Cardiomyocytes can be generated from marrow stromal cells in vitro. J Clin Invest 1999;103:697-705.

29 Kopen GC, Prockop DJ, Phinney DG. Marrow stromal cells migrate throughout forebrain and cerebellum, and they differentiate into astrocytes after injection into neonatal mouse brains. Proc Natl Acad Sci USA 1999;96:10711-10716.

30 Jiang Y, Jahagirdar BN, Reinhardt RL, et al. Pluripotency of mesenchymal stem cells derived from adult marrow. Nature 2002;418:41-49.

31 Reyes M, Dudek A, Jahagirdar B, et al. Origin of endothelial progenitors in human postnatal bone marrow. J Clin Invest 2002;109:337-346.

32 Rajagopol J, Anderson WJ, Kume S, et al. Insulin staining of ES cell progeny from insulin uptake. Science 2003;299:363.

33 Terada N, Hamazaki T, Oka M, et al. Bone marrow cells adopt the phenotype of other cells by spontaneous cell fusion. Nature 2002;416:542-545.

34 Ying QL, Nichols J, Evans EP, et al. Changing potency by spontaneous fusion. Nature 2002;416:545-548.

35 Wang X, Willenbring H, Akkari Y, et al. Cell fusion is the principal source of bone-marrow-derived hepatocytes. Nature 2003;422:897-901.

36 Vassilopoulos G, Wang PR, Russell DW. Transplanted bone marrow regenerates liver by cell fusion. Nature 2003;422:901-904

37 Ianus A, Holz GG, Theise ND, et al. In vivo derivation of glucose-competent pancreatic endocrine cells from bone marrow without evidence of cell fusion. J Clin Invest 2003;111:843-850. 